\title{
Blockade of glycolysis-dependent contraction by oroxylin a via inhibition of lactate dehydrogenase-a in hepatic stellate cells
}

Feixia Wang ${ }^{1}$, Yan Jia ${ }^{1}$, Mengmeng Li ${ }^{1}$, Ling Wang ${ }^{1}$, Jiangjuan Shao ${ }^{2,3}$, Qinglong Guo ${ }^{4}$, Shanzhong Tan ${ }^{5}$, Hai Ding ${ }^{5}$, Anping Chen ${ }^{6}$, Feng Zhang ${ }^{1,2,3^{*}}$ and Shizhong Zheng ${ }^{1,2,3^{*}}$ (i)

\begin{abstract}
Background: Contraction of hepatic stellate cells (HSCs) plays an important role in the pathogenesis of liver fibrosis by regulating sinusoidal blood flow and extracellular matrix remodeling. Here, we investigated how HSC contraction was affected by the natural compound oroxylin A, and elucidated the underlying mechanism.

Methods: Cell contraction and glycolysis were examined in cultured human HSCs and mouse liver fibrosis model upon oroxylin A intervention using diversified cellular and molecular assays, as well as genetic approaches.

Results: Oroxylin A limited HSC contraction associated with inhibiting myosin light chain 2 phosphorylation. Oroxylin A blocked aerobic glycolysis in HSCs evidenced by reduction in glucose uptake and consumption and lactate production. Oroxylin A also decreased extracellular acidification rate and inhibited the expression and activity of glycolysis rate-limiting enzymes (hexose kinase 2, phosphofructokinase 1 and pyruvate kinas type M2) in HSCs. Then, we identified that oroxylin A blockade of aerobic glycolysis contributed to inhibition of HSC contraction. Furthermore, oroxylin A inhibited the expression and activity of lactate dehydrogenase-A ( $\mathrm{LDH}-\mathrm{A})$ in HSCS, which was required for oroxylin A blockade of glycolysis and suppression of contraction. Oral administration of oroxylin A at $40 \mathrm{mg} / \mathrm{kg}$ reduced liver injury and fibrosis, and inhibited HSC glycolysis and contraction in mice with carbon tetrachloride-induced hepatic fibrosis. However, adenovirus-mediated overexpression of LDH-A significantly counteracted the oroxylin A's effects in fibrotic mice.
\end{abstract}

Conclusions: Blockade of aerobic glycolysis by oroxylin A via inhibition of LDH-A reduced HSC contraction and attenuated liver fibrosis, suggesting LDH-A as a promising target for intervention of hepatic fibrosis.

Keywords: Liver fibrosis, Oroxylin a, Hepatic stellate cell, Aerobic glycolysis, Contraction, Lactate dehydrogenase-a

\section{Background}

Hepatic fibrosis is a compensatory repair process in response to a variety of chronic liver injuries. Current paradigm has established that hepatic stellate cells (HSCs) are key effector cells in the initiation and development of hepatic fibrosis [1]. In fibrogenic liver, the quiescent HSCs undergo transdifferentiation into myofibroblasts with high proliferative and migratory capacities, and subsequently

\footnotetext{
*Correspondence: nucmzf@163.com; nytws@163.com

${ }^{1}$ Jiangsu Key Laboratory for Pharmacology and Safety Evaluation of Chinese Materia Medica, School of Pharmacy, Nanjing University of Chinese Medicine, Nanjing 210023, China

Full list of author information is available at the end of the article
}

secrete massive extracellular matrix molecules, accumulating in liver parenchyma and promoting the pathogenesis of hepatic fibrosis [2]. Recent recognition of HSCs as liver-specific pericytes with contractile property is a key milestone in understanding of the biology of these cells [3]. HSCs regulate sinusoidal resistance and blood flow around sinusoids by contraction [4]. In addition, the contractile force generated by HSCs aggravates extracellular matrix remodeling during chronic liver injury [5]. Therefore, elucidating how HSC contraction is regulated may facilitate the development of therapeutic strategies for chronic liver disease.

(c) The Author(s). 2019 Open Access This article is distributed under the terms of the Creative Commons Attribution 4.0 International License (http://creativecommons.org/licenses/by/4.0/), which permits unrestricted use, distribution, and reproduction in any medium, provided you give appropriate credit to the original author(s) and the source, provide a link to the Creative Commons license, and indicate if changes were made. The Creative Commons Public Domain Dedication waiver (http://creativecommons.org/publicdomain/zero/1.0/) applies to the data made available in this article, unless otherwise stated. 
Cell contraction involves dynamic synthesis and decomposition of actin and formation of large cytoskeletal structures [6]. When cells contract, the myosin cross-bridge periodically binds to actin, which dissociates and hydrolyzes ATP, releasing energy for actin filaments [7]. Cell contraction is thus a highly energy-consuming process. It has been well established that a key metabolic hallmark of cancer cells is aerobic glycolysis, termed Warburg effect [8]. Although glycolysis produces less ATP than oxidative phosphorylation does, the Warburg effect favors cell growth by rapidly providing ATP and carbon sources [8]. Glycolysis has some rate-limiting enzymes including hexokinase 2 (HK2), phosphofructokinase 1 (PFK1) and pyruvate kinas type M2 (PKM2) successively, converting glucose to pyruvate [9]. Notably, final conversion of pyruvate to lactate is a crucial step catalyzed by lactate dehydrogenase (LDH), of which LDH-A is a major subtype [10]. High expression or activity of LDH-A allows for rapid glycolysis flux so as to meet the energy demands of rapidly proliferating cells [11]. Recent evidence suggests that the activated HSCs are similar to the highly proliferative cancer cells with regard to their biosynthetic and bioenergetic requirements [12]. Aerobic glycolysis is a striking metabolic phenotype of activated HSCs during liver fibrosis [12]. However, little is known about the role of aerobic glycolysis in controlling of HSC contraction.

Natural products have been an importance source of drug candidates nowadays. Oroxylin A is an attractive natural compound with promising pharmacological activities. For example, oroxylin A was found to inhibit the growth and proliferation of hepatoma cells [13, 14]. Oroxylin A could also reduce glucose uptake and lactate production in HepG2 cells under hypoxia [15], and inhibit glycolysis-dependent growth of human breast tumors [16]. Our previous studies demonstrated that oroxylin A reduced liver fibrosis associated with induction of HSC autophagy [17]. We hold that the mechanisms underlying the antifibrotic effects of oroxylin A have not been fully understood. Here, we investigated whether and how oroxylin A affected HSC contraction with a focus on the association of aerobic glycolysis.

\section{Methods}

\section{Chemicals and antibodies}

Oroxylin A (HPLC purity 99.9\%) was kindly provided by Professor Qinglong Guo (China Pharmaceutical University, Nanjing, China). Compounds 2-deoxy-D-glucose (2-DG) and galloflavin were purchased from Apexbio Technology (Houston, TX, USA). These reagents were dissolved with dimethylsulfoxide at indicated concentrations for in vitro experiments. The following primary antibodies were used for Western blot analysis in current study: antibodies against HK2, PFK1, PKM2, LDH-A, $\beta$-actin and GAPDH were obtained from Proteintech Group (Chicago, IL, USA); antibodies against $\mathrm{p}-\mathrm{MLC} 22^{\mathrm{Ser} 19}, \mathrm{MLC} 2, \alpha-\mathrm{SMA}$, fibronectin and $\alpha 1(\mathrm{I})$ procollagen were obtained from Cell Signaling Technology (Danvers, MA, USA). Horseradish peroxidase-conjugated anti-mouse and anti-rabbit secondary antibodies were obtained from Proteintech Group (Chicago, IL, USA).

\section{Cell culture and transfection}

Human HSC line LX2 cells and human normal hepatocyte line LO2 cells were obtained from the Cell Bank of Chinese Academy of Sciences (Shanghai, China). Cells were characterized using human short tandem repeat markers. Cells were cultured in Dulbecco's modified eagle medium (Invitrogen, Grand Island, NY, USA) with $10 \%$ fetal bovine serum (Wisent Biotechnology Co., Ltd., Nanjing, China), $1 \%$ antibiotics, and grown in a $5 \% \mathrm{CO}_{2}$ humidified atmosphere at $37^{\circ} \mathrm{C}$. LDH-A siRNA (sc-43,893) and control siRNA (sc-37,007) were obtained from Santa Cruz Biotechnology (Santa Cruz, CA, USA). LDH-A overexpression plasmid pcDNA3.1(+)-LDH-A was constructed by Jiangsu KeyGEN Biotechnology Co. Ltd. (Nanjing, China). Transfection with LDH-A siRNA or overexpression plasmid was performed using the Lipofectamine 2000 Transfection Reagent (Life Technologies, Grand Island, NY, USA) according to the protocols provided by the manufacturer.

\section{Collagen gel contraction assay}

Collagen gel contraction assays were performed as we previously described [18]. Percentages of original gel area were quantified using the Image J software (Media Cybernetics, Rockville, MD, USA). Representative views are shown.

\section{Cytoskeleton staining}

Cytoskeleton was visualized using FITC-conjugated phalloidin (Beyotime Biotechnology, Haimen, China) according to our previously described methods [18]. The nuclei of cells were stained with diamidino-phenyl-indole (DAPI). Photographs were blindly taken at five random fields under a microscope (ZEISS Axio vert. A1, Germany). Representative views are shown.

\section{Immunofluorescence staining}

Staining with LX2 cells or mouse liver tissues was performed according to our descried methods [19]. The nuclei of cells were stained with DAPI. Photographs were blindly taken at five random fields under a microscope (ZEISS Axio vert. A1, Germany). Representative views are shown.

\section{Glucose uptake assay}

The glucose uptake by LX2 cells was determined using a Glucose Uptake Assay Kit (Abnova, Taiwan, China) according to the manufacturer's instructions. In this assay, the glucose analog 2-DG is metabolized to 2-DG-6-phosphate, which is proportional to glucose 
uptake by cells. The accumulated 2-DG-6-phosphate is enzymatically coupled to generate NADPH, which is specifically monitored by a NADPH sensor. The signal can be read by an absorbance microplate reader by reading the OD ratio at wavelength 570 to $610 \mathrm{~nm}$.

\section{Glucose consumption assay}

The glucose consumption by LX2 cells was determined using an enzyme-linked immunosorbent assay kit (Shanghai Meilian Biology Technology Co. Ltd., Shanghai, China) for measuring glucose oxidase (GOD) activity according to the protocols provided by the manufacture. GOD is an endogenous oxido-reductase efficiently catalyzing the oxidization of glucose into gluconic acid. Its activity is an alternative indicator of glucose consumption [20].

\section{Measurement of lactate levels}

Lactate levels in lysates of LX2 cells or mouse liver tissues were measured using kits (Nanjing Jiancheng Bioengineering Institute, Nanjing, China) following the manufacturer's instructions.

\section{Measurement of extracellular acidification rate (ECAR)}

ECAR was measured using a pH-Xtra ${ }^{\mathrm{ma}}$ Glycolysis Assay Kit from Luxcel Biosciences (Cork, Ireland) following the manufacturer's instructions and reported methods [21]. The $\mathrm{pH}-\mathrm{Xtra}^{\mathrm{Tm}}$ assay uses a $\mathrm{pH}$-sensitive fluorophore which detects acidification due to glycolysis-related release of lactate.

\section{Measurement of intracellular ATP levels}

Intracellular ATP levels were determined using an ATP Assay Kit provided by Beyotime Institute of Biotechnology (Haimen, China) according to the protocols provided by the manufacture.

\section{Enzyme activity assay}

The intracellular activities of HK2, PFK1, PKM2 and LDH-A in LX2 cell were measured using kits (Shanghai Meilian Biology Technology Co. Ltd., Shanghai, China) according to the protocols provided by the manufacture.

\section{Cell viability assay}

The viability of LX2 cells or LO2 cells treated with 2-DG or galloflavin was evaluated using MTT assays. Briefly, the medium of treated cells was replaced with $100 \mu \mathrm{l}$ phosphate buffered saline containing $0.5 \mathrm{mg} / \mathrm{ml}$ MTT and then was incubated at $37^{\circ} \mathrm{C}$ for $4 \mathrm{~h}$. The crystals were dissolved with $200 \mu \mathrm{l}$ dimethylsulfoxide. The spectrophotometric absorbance at $490 \mathrm{~nm}$ was measured by a SPECTRAmax ${ }^{\text {Tix }}$ microplate spectrophotometer (Molecular Devices, Sunnyvale, CA, USA). Cell viability was expressed as percentage of control.

\section{Human liver samples}

Liver samples from five healthy subjects and five patients with liver fibrosis were provided by the Nanjing Hospital Affiliated to Nanjing University of Chinese Medicine (Nanjing, China). The study followed the tenets of the Declaration of Helsinki, and informed written consents were obtained from all patients followed by explanation of the nature and possible consequences of the study. The study protocol was approved by the Medical Ethical Committee of the Second Hospital of Nanjing.

\section{Animal procedures and treatments}

Animal experimental procedures were approved by the Institutional and Local Committee on the Care and Use of Animals of Nanjing University of Chinese Medicine, and all animals were received humane care according to the National Institutes of Health (USA) guidelines. Thirty male ICR mice (8-week old) were obtained from Shanghai Slac Laboratory Animal Co., Ltd. (Shanghai, China). Mice were housed in standardized conditions at $20 \pm 2{ }^{\circ} \mathrm{C}$ room temperature, $40 \pm 5 \%$ relative humidity and a $12 \mathrm{~h}$ light/ dark cycle. A mixture of carbon tetrachloride $\left(\mathrm{CCl}_{4}\right)$ and olive oil $[2: 3(\mathrm{v} / \mathrm{v})]$ was used to induce hepatic fibrosis in mice via intraperitoneal injection $(0.1 \mathrm{ml} / 100 \mathrm{~g}$ body weight). Thirty mice were randomly divided into five groups $(n=6)$ : (1) control, (2) model, (3) oroxylin A treatment, (4) oroxylin A treatment plus adenovirus vector, and (5) oroxylin A treatment plus LDH-A plasmid adenovirus (constructed by OBiO Technology Co. Ltd., Shanghai, China). Initially, mice in groups 4 and 5 were received caudal vein injection with corresponding adenovirus once. Two weeks later, mice in groups $2-5$ were received intraperitoneal injection with $\mathrm{CCl}_{4}$ every three days for 4 weeks. Simultaneously, mice in groups 3-5 were orally given oroxylin A suspended in CMC-Na solution at 40 $\mathrm{mg} / \mathrm{kg}$ once daily for 4 weeks. This dose was determined by preliminary experiments. Mice in group 1 were orally given equal amount of $\mathrm{CMC}-\mathrm{Na}$ solution once daily and injected with olive oil intraperitoneally every three days for 4 weeks, and mice in group 2 were also orally given equal amount of CMC-Na solution once daily for 4 weeks. At the end of experiments, all mice were anesthetized with isoflurane followed by blood collection via retro orbital sinus and isolation of liver.

\section{Serum biochemistry}

Serum levels of alanine aminotransferase (ALT), aspartate aminotransferase (AST), total bilirubin (TBIL), indirect bilirubin (IBIL), hyaluronic acid (HA), laminin (LN) and procollagen type III (PC-III) were measured using kits (Nanjing Jinting Bioengineering Institute, Nanjing, China) according to the protocols provided by the manufacture. 


\section{Measurement of hepatic hydroxyproline (Hyp)}

The Hyp levels in mouse liver tissues were measured using a kit (Nanjing Jiancheng Bioengineering Institute, Nanjing, China) according to the protocols provided by the manufacture.

\section{Liver histopathology and collagen staining}

Mouse liver tissues were fixed in 10\% neutral buffered formalin and embedded in paraffin. Hematoxylin-eosin $(H \& E)$ staining was used for assessment of histopathology according to standard methods. Masson staining and Sirius Red staining were used for exanimation of collagens according to standard methods. Photographs were blindly taken at five random fields under a microscope (ZEISS Axio vert. A1, Germany). Representative views are shown.

\section{Immunohistochemistry}

Mouse liver tissue sections were incubated with primary antibody against $\alpha$-smooth muscle actin ( $\alpha$-SMA) for immunohistochemical evaluation using standard methods. Photographs were blindly taken at five random fields under a microscope (ZEISS Axio vert. A1, Germany). Representative views are shown.

\section{Scanning electronic microscopy (SEM)}

Sinusoidal fenestration of mouse liver was examined by SEM according to our previously reported methods [19]. Photographs were blindly taken at five random fields, and representative images are shown.

\section{Real-time PCR}

Total RNA was extracted from LX2 cells, mouse liver tissues, or human liver samples using Trizol reagent (Sigma, Saint Louis, MO, USA). Total RNA was subject to reverse transcription to cDNA using the TransScript All-in One First-Strand cDNA Synthesis SuperMix for qPCR (One-Step gDNA Removal) Kits provided by TransGen Biotech Co., Ltd. (Beijing, China) according to the protocols. Real-time PCR was performed using the SYBR Green Master Mix (Vazyme Biotech Co., Ltd., Nanjing China) according to the protocol. Fold changes in the mRNA levels of target genes were related to the invariant control glyceraldehyde phosphate dehydrogenase (GAPDH). The primers (GenScript Co., Ltd., Nanjing, China) are listed in Additional file 1: Table S1.

\section{Western blot assay}

Whole cell protein extracts were prepared from LX2 cells or mouse liver tissues with RIPA buffer containing protease inhibitors and phosphatase inhibitors. Protein detection and band visualization and quantification were performed as we previously described [22]. $\beta$-Actin or
GAPDH was used as an invariant control for equal loading of total proteins. Representative blots were shown.

\section{Statistical analysis}

Data from at least triplicate experiments are presented as mean \pm SD. One-way ANOVA was performed to analyze the data using GraphPad Prism 7 (San Diego, CA, USA). In all cases, a $P$ value of 0.05 or lower was considered significant.

\section{Results \\ Oroxylin a inhibits HSC contraction}

Results of collagen gel contraction assays showed that oroxylin A concentration-dependently inhibited HSC contraction, and that oroxylin $\mathrm{A}$ at 30 and $40 \mu \mathrm{M}$ produced significant effects (Fig. 1a). The organization of cell contractile machinery can be manifested by cytoskeleton arrangement [23]. Cytoskeleton fluorescence staining revealed that oroxylin A reduced the formation of actin stress fibers and disturbed the microfilament skeleton in a concentration-dependent manner in HSCs (Fig. 1b). Phosphorylation of myosin light chain 2 (MLC2) is an important event during cell contraction [24]. Western blot analyses demonstrated that oroxylin A concentration-dependently decreased MLC2 phosphorylation in HSCs (Fig. 1c). Immunofluorescence analyses of MLC2 phosphorylation provided consistent results (Fig. 1d). Together, these data indicated that oroxylin A inhibited HSC contraction.

\section{Oroxylin a blocks aerobic glycolysis leading to inhibition of HSC contraction}

We then tested the effects of oroxylin A on aerobic glycolysis of HSCs. We observed that oroxylin A decreased glucose uptake (Fig. 2a) and glucose consumption indicated by GOD activity (Fig. 2b) in a concentration-dependent fashion in HSCs. Lactate production and ECAR were also reduced by oroxylin A concentration-dependently (Fig. 2c, d). We further detected the effects of oroxylin $\mathrm{A}$ on three rate-limiting enzymes HK2, PFK1 and PKM2, and observed that the mRNA and protein expression of these enzymes were downregulated by oroxylin A in HSCs (Fig. 2e, f). Meanwhile, oroxylin A decreased the intracellular activities of HK2, PFK1 and PKM2 (Fig. 2g). Additional data showed that the intracellular ATP levels were reduced by oroxylin A concentration-dependently in HSCs (Additional file 2: Figure S1). The above findings collectively revealed that the overall glycolytic flux and activity were effectively blocked by oroxylin A, cutting of the energy supply within HSCs.

We next asked whether blockade of aerobic glycolysis was associated with the reduced contractile capacity in oroxylin A-treated HSCs. The glycolysis inhibitor 2-DG was used to test the association. We used 2-DG at $5 \mathrm{mM}$ for experiments based on the observation that 2-DG at this 

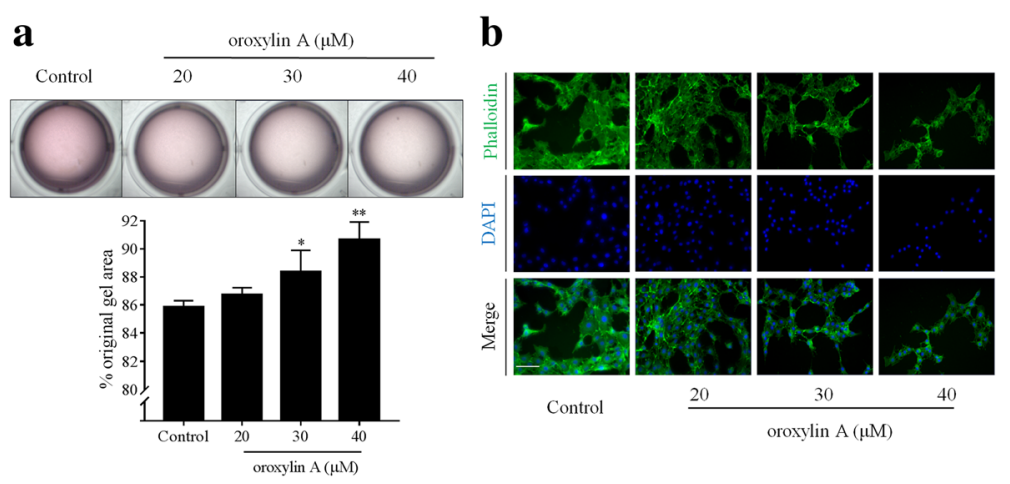

$\mathbf{C}$
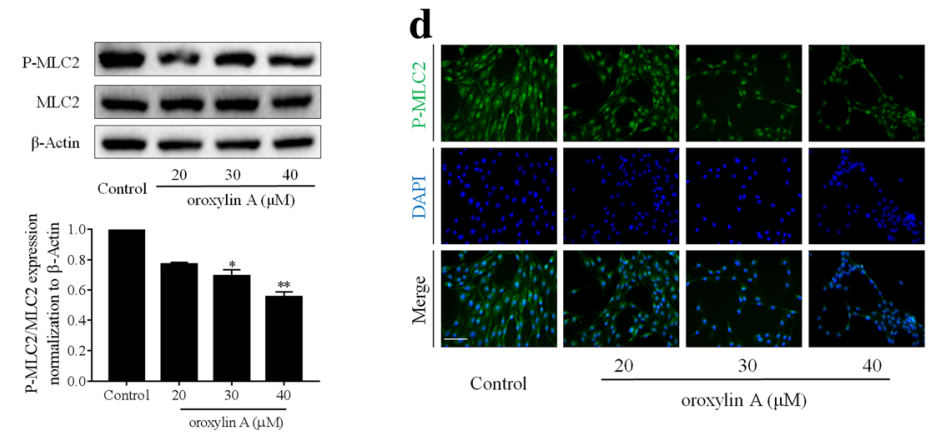

Fig. 1 Oroxylin A inhibits HSC contraction. LX2 cells were treated with oroxylin A at indicated concentrations for $24 \mathrm{~h}$. a Collagen gel contraction assays with quantification. b Cytoskeleton fluorescence staining, scale bar: $20 \mu \mathrm{m}$. c Western blot analyses of MLC2 phosphorylation with quantification. $\mathbf{d}$ Immunofluorescence analyses of MLC2 phosphorylation, scale bar: $20 \mu \mathrm{m}$. For statistical significance of this figure: ${ }^{*} p<0.05$ vs. control, ${ }^{* *} p<0.01$ vs. control
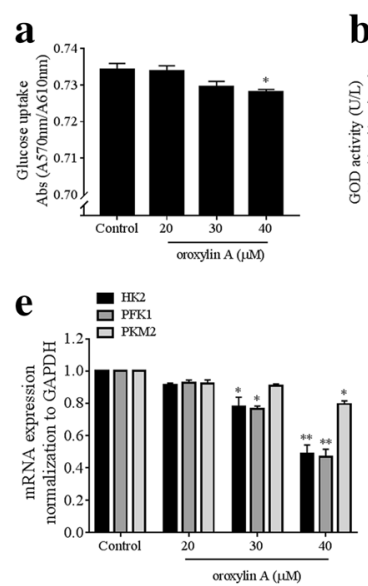

g

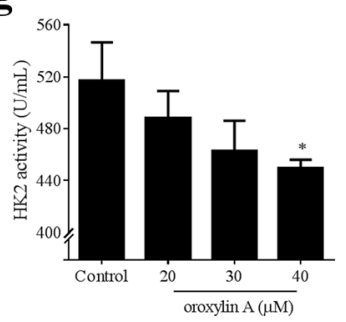

b

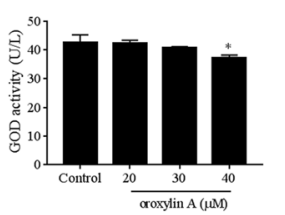

f
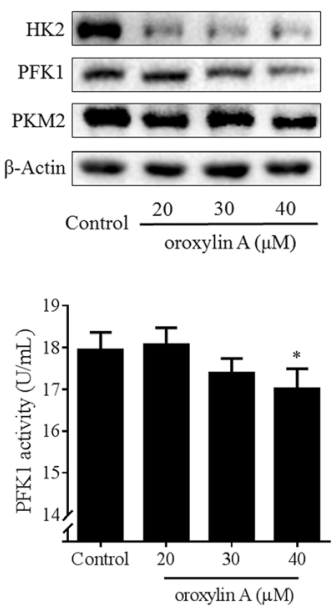

d
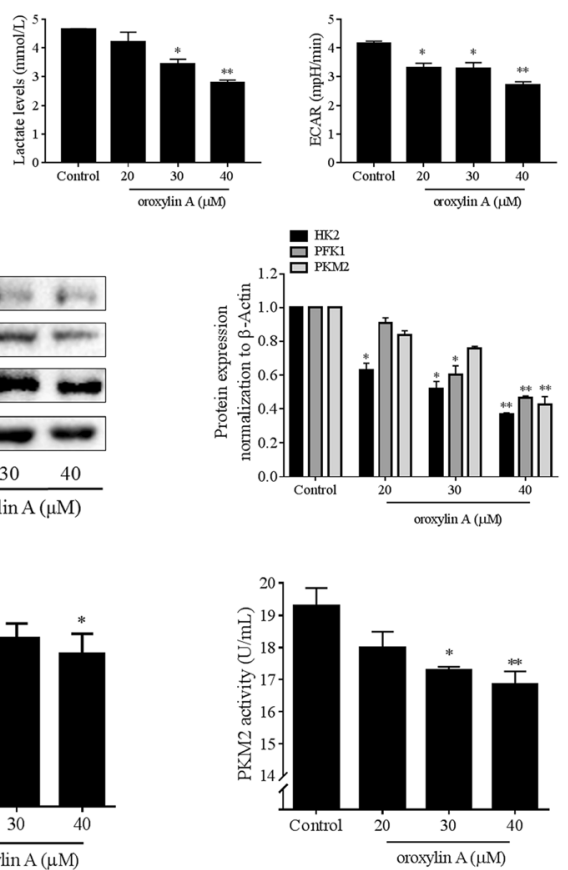

Fig. 2 Oroxylin A blocks aerobic glycolysis in HSCs. LX2 cells were treated with oroxylin A at indicated concentrations for 24 h. a Measurements of glucose uptake. b Measurements of glucose consumption indicated by GOD activity. c Measurements of intracellular lactate levels. d Measurements of ECAR. e Real-time PCR analyses of mRNA expression of HK2, PFK1 and PKM2. f Western blot analyses of protein expression of HK2, PFK1 and PKM2 with quantification. g Measurements of intracellular enzyme activities of HK2, PFK1 and PKM2. For statistical significance of this figure: ${ }^{*} p<0.05$ vs. control, ${ }^{* *} p<0.01$ vs. control 
concentration suppressed HSC viability but did not affect hepatocyte viability (Additional file 3: Figure S2a, b). Collagen gel contraction assays showed that 2-DG at $5 \mathrm{mM}$, similar to oroxylin A at $40 \mu \mathrm{M}$, significantly suppressed HSC contraction, and their combination produced more potent inhibitory effects (Fig. 3a). Cytoskeleton fluorescence staining revealed that microfilament skeleton was disrupted by 2-DG and its combination with oroxylin A (Fig. 3b). Examinations of MLC2 phosphorylation using Western blot analysis and immunofluorescence staining consistently exhibited that 2-DG at $5 \mathrm{mM}$ alone, or combined with oroxylin A at $40 \mu \mathrm{M}$, significantly downregulated the phosphorylation levels of MLC2 in HSCs (Fig. 3c, d). Altogether, these results indicated that blockade of aerobic glycolysis by oroxylin A resulted in the suppression of HSC contraction.

\section{Oroxylin a inhibits LDH-A in HSCs}

Given that LDH-A is a central player in glycolysis and has a multifunctional role in cell biology [25], we next focused on the regulation of LDH-A by oroxylin A in HSCs. We observed that the mRNA levels of LDH-A in liver tissues from patients with hepatic fibrosis were significantly higher than that in healthy liver tissues (Additional file 4: Figure S3), strongly suggesting the role of LDH-A in the pathology of liver fibrosis. We then found that the LDH-A mRNA expression was reduced by oroxylin $\mathrm{A}$ in a concentration-dependent manner in cultured HSCs (Fig. 4a). Oroxylin A also downregulated the protein abundance of LDH-A in HSCs evidenced by Western blot and immunofluorescence analyses (Fig. 4b, c). Consistently, the intracellular enzyme activity of LDH-A was decreased by oroxylin A concentration-dependently (Fig. 4d). Taken together, these results revealed that oroxylin A inhibited the expression and activity of LDH-A in HSCs.

\section{Inhibition of LDH-A is required for oroxylin a to block} aerobic glycolysis and reduce contraction in HSCs

The above results suggested LDH-A as a potential target molecule for oroxylin A in HSCs, and we subsequently attempted to confirm this hypothesis. The compound galloflavin, a selective pharmacological inhibitor of LDH-A [26], was used to test the role of LDH-A in oroxylin A blockade of aerobic glycolysis. We used galloflavin at $20 \mu \mathrm{M}$ for experiments based on the observation that galloflavin at this concentration inhibited HSC viability but did not affect hepatocyte viability (Additional file 3: Figure S2c, d). We observed that galloflavin at $20 \mu \mathrm{M}$, similar to oroxylin A at $40 \mu \mathrm{M}$, significantly inhibited glucose uptake and consumption and reduced the production of lactate in HSCs, and that combination of galloflavin and oroxylin A produced more potent reducing effects on these parameters (Fig. 5a-c). Further examinations of glycolysis rate-limiting enzymes showed that galloflavin at $20 \mu \mathrm{M}$ significantly decreased the expression and activities of HK2, PFK1 and

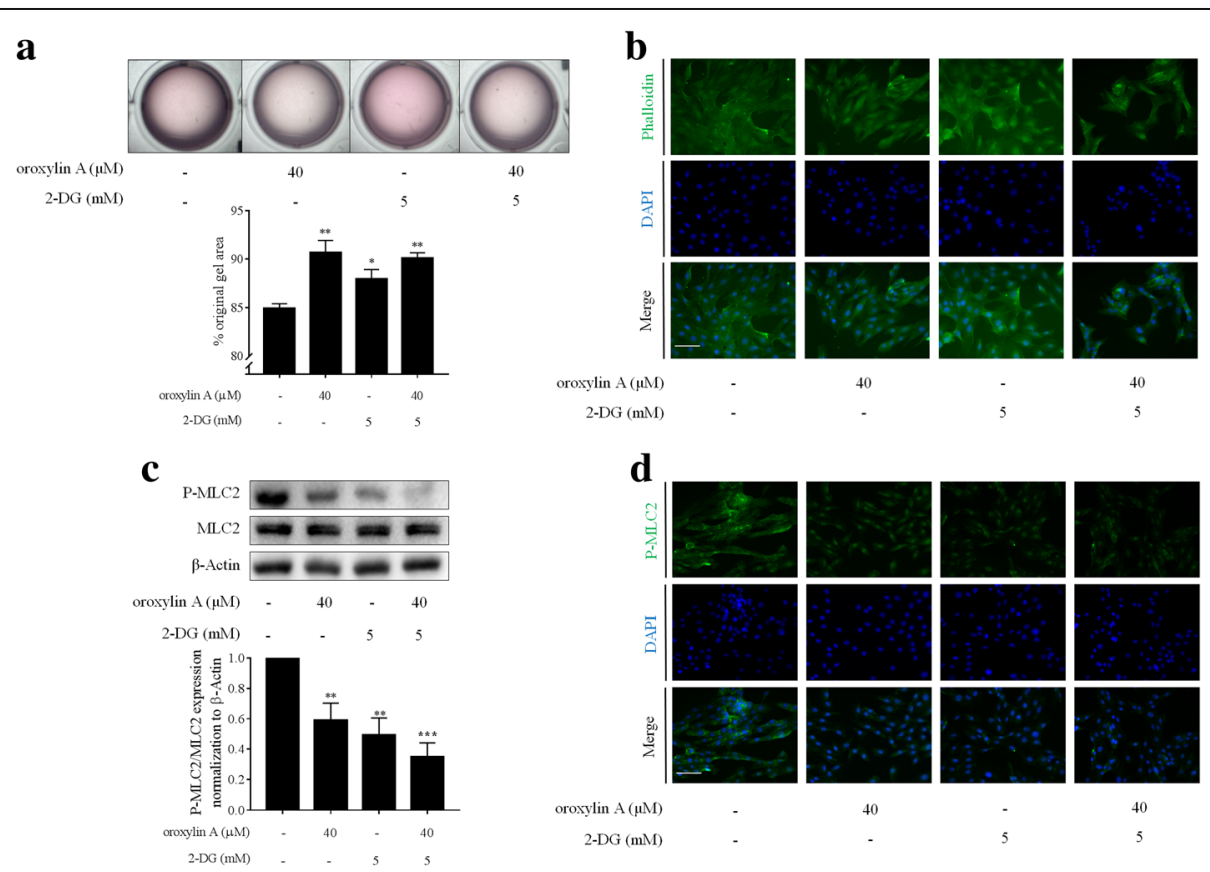

Fig. 3 Oroxylin A blockade of aerobic glycolysis led to inhibition of HSC contraction. LX2 cells were treated with oroxylin A and/or 2-DG at indicated concentrations for $24 \mathrm{~h}$. a Collagen gel contraction assays with quantification. b Cytoskeleton fluorescence staining, scale bar: $20 \mu \mathrm{m}$. $\mathbf{c}$ Western blot analyses of MLC2 phosphorylation with quantification. $\mathbf{d}$ Immunofluorescence analyses of MLC2 phosphorylation, scale bar: $20 \mu \mathrm{m}$. For statistical significance of this figure: ${ }^{*} p<0.05$ vs. control, ${ }^{* *} p<0.01$ vs. control, ${ }^{* * *} p<0.001$ vs. control 

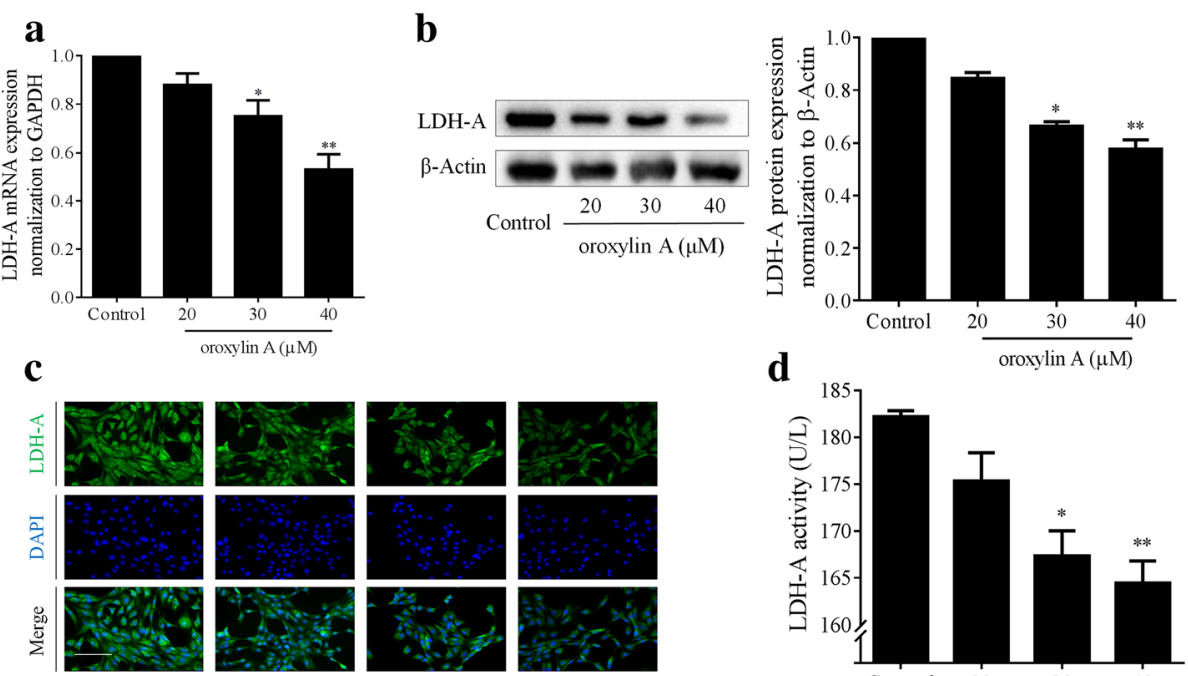

d

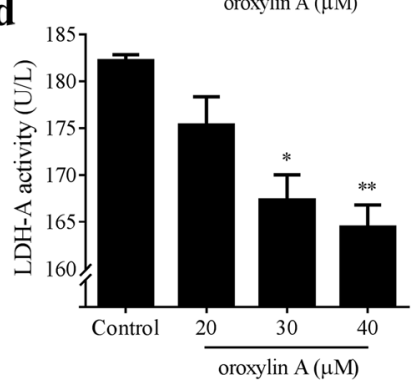

Fig. 4 Oroxylin A inhibits LDH-A in HSCs. LX2 cells were treated with oroxylin A at indicated concentrations for $24 \mathrm{~h}$. a Real-time PCR analyses of mRNA expression of LDH-A. $\mathbf{b}$ Western blot analyses of protein expression of $\mathrm{LDH}-\mathrm{A}$ with quantification. $\mathbf{c}$ Immunofluorescence analyses of $\mathrm{LDH}$ A expression. d Measurements of intracellular LDH-A activity. For statistical significance of this figure: ${ }^{*} p<0.05$ vs. control, ${ }^{* *} p<0.01$ vs. control

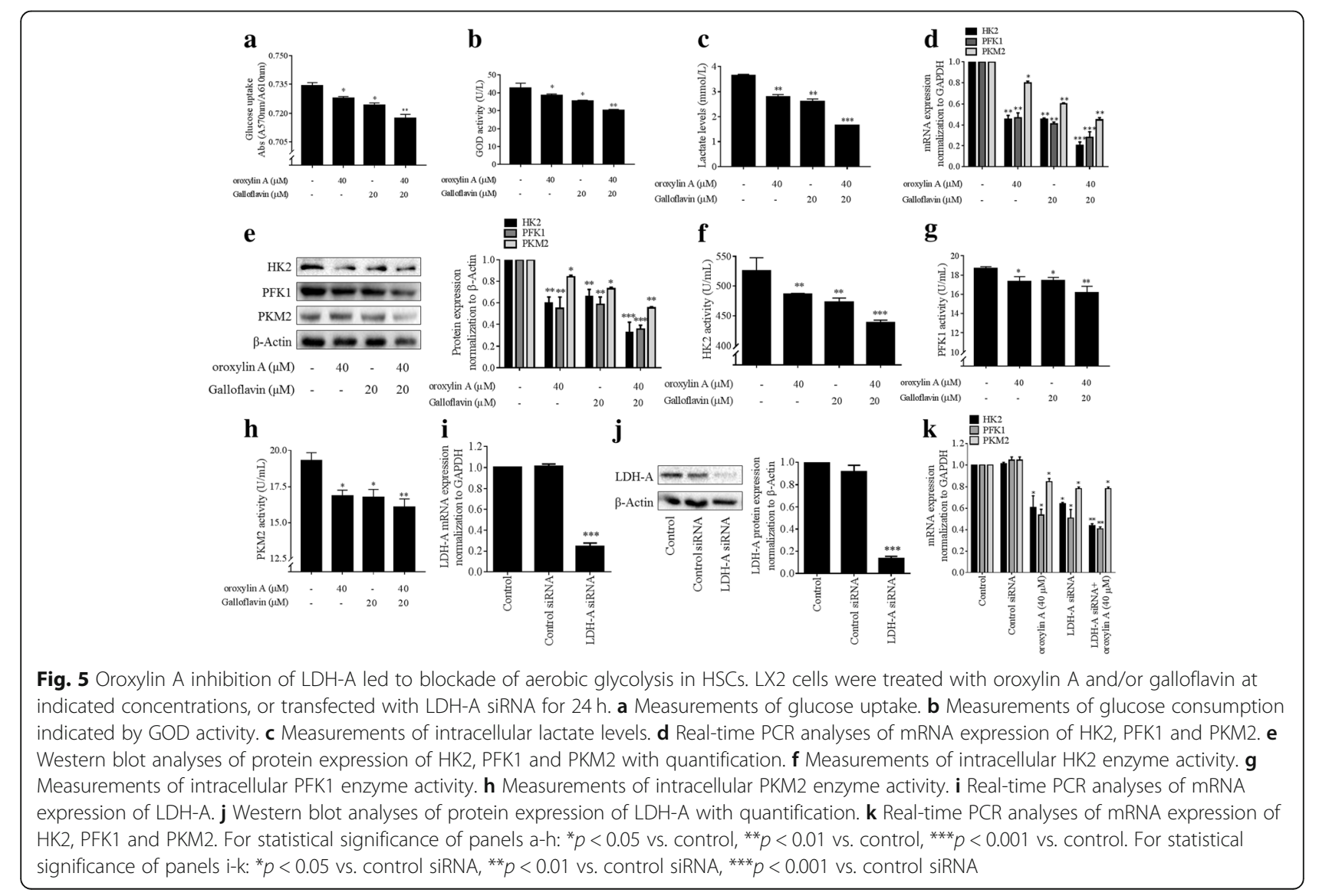


PKM2 in HSCs, and that its combination with oroxylin A resulted in more potent inhibitory effects on these enzymes (Fig. 5d-h). To confirm the results, HSCs were transfected with LDH-A siRNA to knockdown LDH-A expression at both mRNA and protein levels (Fig. 5i, j). Consistently, transfection with LDH-A siRNA alone or combined with oroxylin A treatment significantly downregulated the mRNA expression of HK2, PFK1 and PKM2 (Fig. 5k). Additionally, overexpression of LDH-A increased the expression of HK2, PFk1 and PKM2 and rescued oroxylin A-induced reduction of these enzymes in HSCs (Additional file 5: Figure S4). Overall, genetic deficiency of LDH-A or pharmacological inhibition of LDH-A effectively diminished the glycolytic activity in HSCs, and synergistic effects could be achieved when combined with oroxylin A, suggesting that inhibition of LDH-A was required for oroxylin A to block aerobic glycolysis.

We further investigated the association between inhibition of LDH-A and suppression of contraction by oroxylin A in HSCs. As expected, collagen gel contraction assays and cytoskeleton fluorescence staining showed that galloflavin at $20 \mu \mathrm{M}$, similar to oroxylin $\mathrm{A}$ at $40 \mu \mathrm{M}$, significantly reduced HSC contraction, and that combination of the two compounds produced more potent effects (Fig. 6a, b), which were confirmed by analyses of MLC2 phosphorylation by Western blot and immunofluorescence assays (Fig. 6c, d). To validate the results, siRNA-mediated knockdown of LDH-A was performed in HSCs. The obtained data exhibited that transfection with LDH-A siRNA, similar to oroxylin A treatment alone, apparently disrupted the microfilament skeleton evidenced by cytoskeleton fluorescence staining (Fig. 6e) and reduced MLC2 phosphorylation demonstrated by Western blot assays (Fig. 6f). Additionally, overexpression of LDH-A promoted dense arrangement of cytoskeleton and increased MLC2 phosphorylation, and considerably abrogated oroxylin A-inhibited HSC contraction (Additional file 6: Figure S5). Collectively, these discoveries indicated that inhibition of LDH-A was required for oroxylin A reduction of HSC contraction.
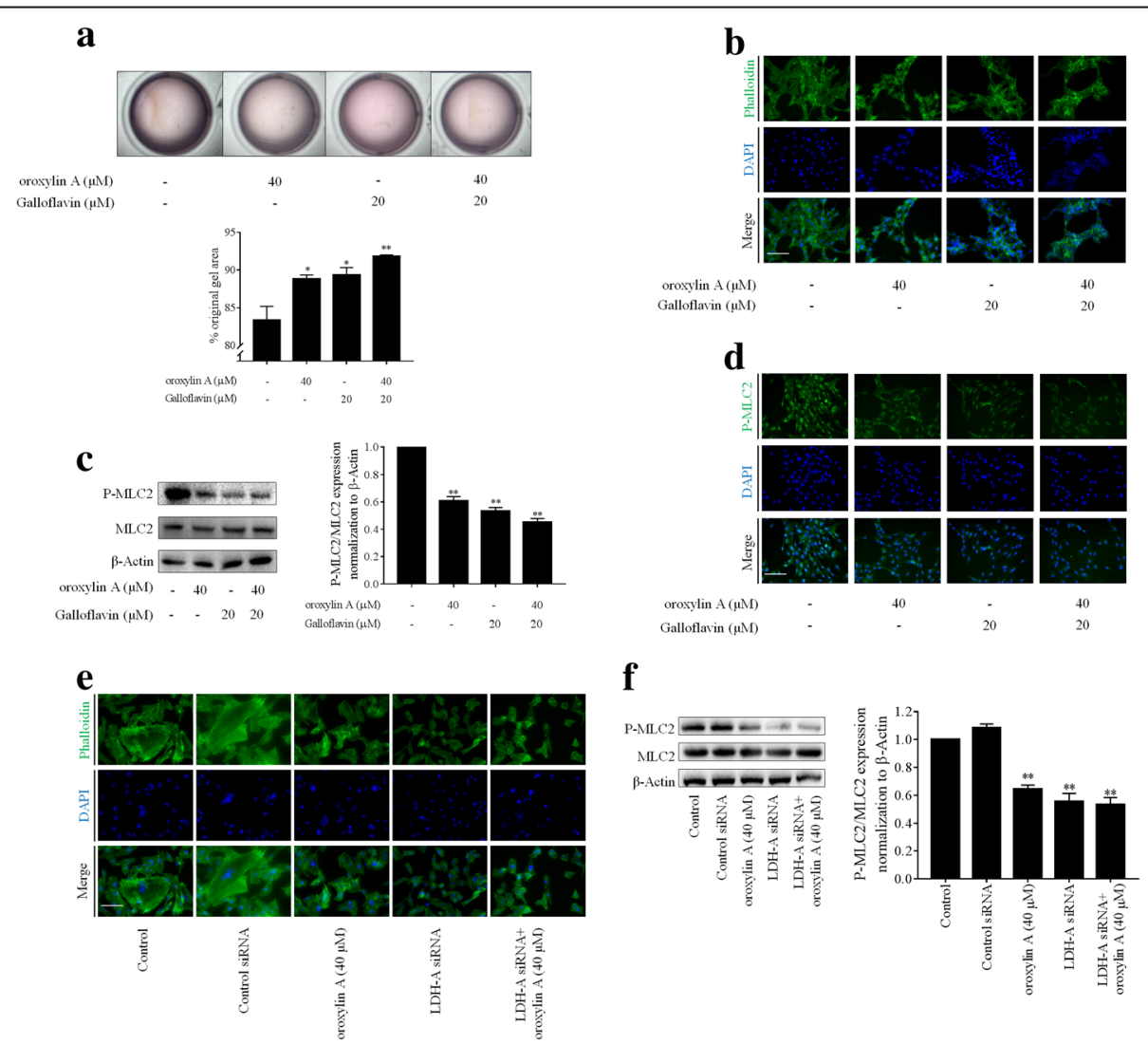

Fig. 6 Oroxylin A inhibition of LDH-A led to reduction of HSC contraction. LX2 cells were treated with oroxylin A and/or galloflavin at indicated concentrations, or transfected with LDH-A siRNA for $24 \mathrm{~h}$. a Collagen gel contraction assays with quantification. b Cytoskeleton fluorescence staining, scale bar: $20 \mu \mathrm{m}$. c Western blot analyses of MLC2 phosphorylation with quantification. d Immunofluorescence analyses of MLC2 phosphorylation, scale bar: $20 \mu \mathrm{m}$. e Cytoskeleton fluorescence staining, scale bar: $20 \mu \mathrm{m}$. f Western blot analyses of MLC2 phosphorylation with quantification. For statistical significance of panels a-d: ${ }^{*} p<0.05 \mathrm{vs}$. control, ${ }^{* *} p<0.01 \mathrm{vs}$. control. For statistical significance of panels e-f: ${ }^{* *} p<0.01 \mathrm{vs}$. control siRNA 
Oroxylin a alleviates liver fibrotic injury and inhibits HSC glycolysis and contraction by targeting LDH-A in mice intoxicated with $\mathrm{CCl}_{4}$

We used the classical liver fibrosis model induced by intraperitoneal injection of $\mathrm{CCl}_{4}$ in mice to establish the in vivo relevance of the above culture-system findings. Because our recent studies have clearly demonstrated that oroxylin A had potent in vivo antifibrotic effects [17, 27], we here focused on testifying whether the effects of oroxylin A were dependent on regulation of LDH-A using adenovirus-mediated overexpression of LDH-A in mice. Oroxylin A reduced the liver/body weight ratio and downregulated the serum levels of hepatocyte injury markers (ALT, AST, TBIL, and IBIL) in fibrotic mice, but these effects of oroxylin A were abolished by overexpression of LDH-A (Fig. 7a-c). Similar changes were observed in the measurements of serum levels of fibrotic markers (HA, LN, and PC-III) and hepatic Hyp contents (Fig. 7d, e). Histological assessments and collagen staining assays showed that oroxylin A amelioration of hepatic structure and collagen deposition was abolished by overexpression of LDH-A in vivo (Fig. 7f). We then examined HSC activation markers and found that oroxylin A significantly reduced the expression of $\alpha$-SMA, fibronectin and $\alpha 1(\mathrm{I})$ procollagen at both mRNA and protein levels in mouse fibrotic liver, but these effects were counteracted by overexpression of LDH-A in fibrotic mice (Fig. 7f-h). Interestingly, SEM data exhibited that treatment with oroxylin A inhibited sinusoidal capillarization and restored the fenestrae of liver sinusoidal endothelial cells in fibrotic mice, but overexpression of LDH-A diminished oroxylin A improvement of hepatic vascular architecture during liver fibrogenesis (Fig. 7i). Altogether, these observations indicated that oroxylin A alleviated liver fibrotic injury by targeting LDH-A in mice.

We subsequently evaluated the effects of oroxylin A on HSC glycolysis and contraction in fibrotic mice. We observed that hepatic lactate levels in fibrosis model mice were significantly elevated compared to that of control mice, and that oroxylin A intervention considerably decreased hepatic lactate levels, which was abrogated by overexpression of LDH-A (Fig. 8a). Treatment with oroxylin A downregulated the mRNA and protein expression of HK2, PFK1, PKM2 and LDH-A in mouse fibrotic liver, but their reduction was remarkably rescued by overexpression of LDH-A (Fig. 8b, c). Further immunofluorescence analyses with $\alpha$-SMA staining for indicating HSCs revealed that these key glycolysis rate-limiting enzymes

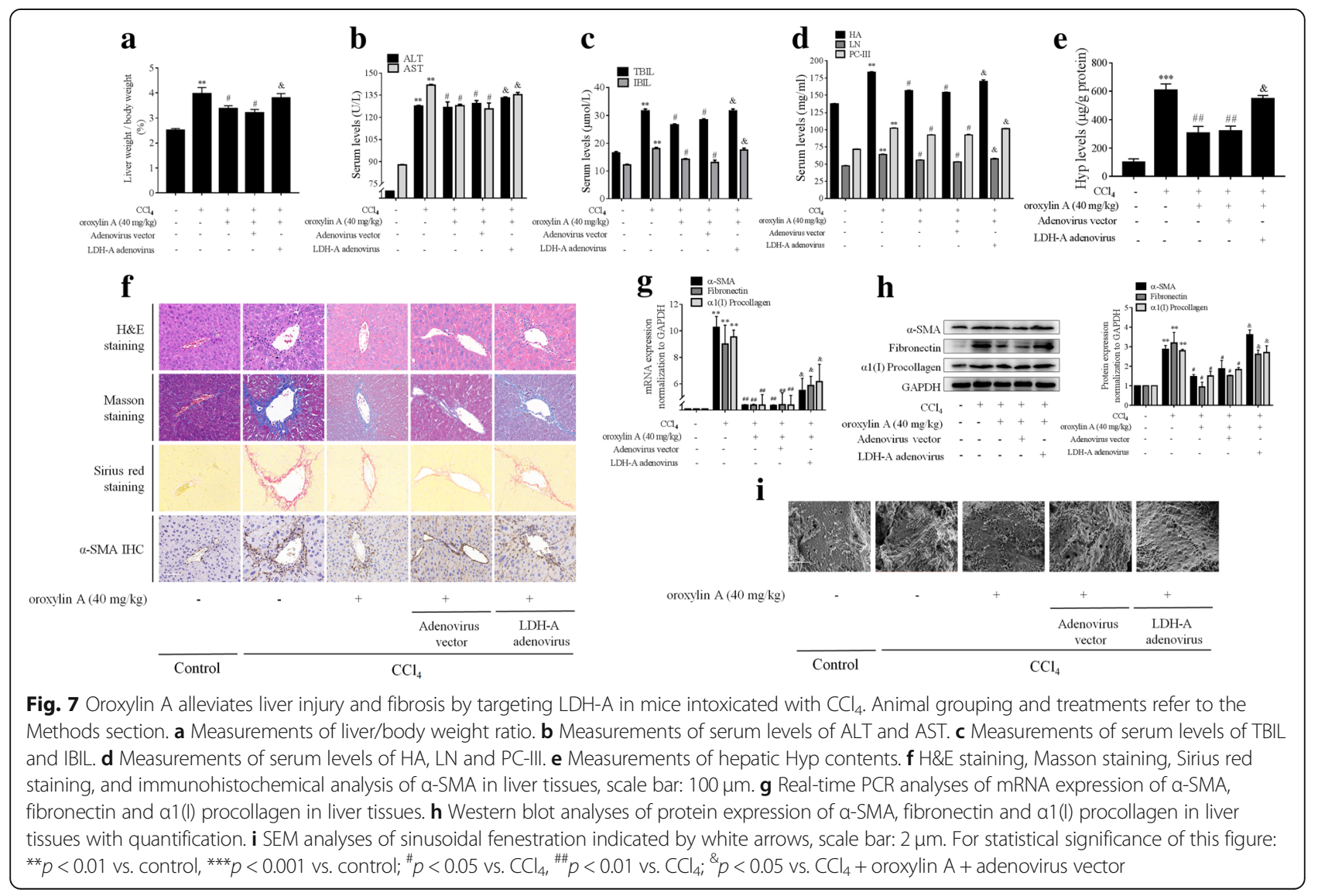




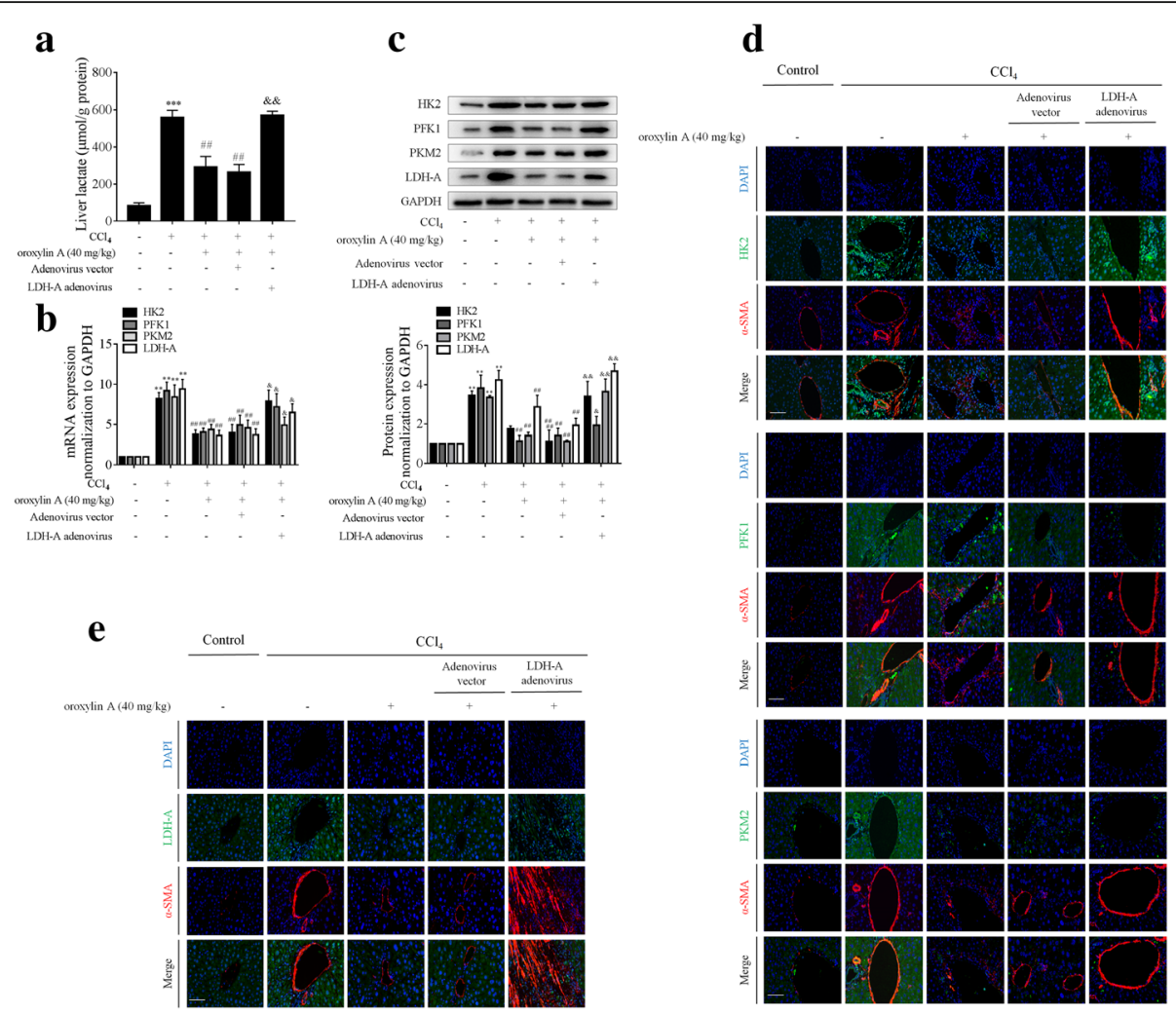

Fig. 8 Oroxylin A inhibits HSC glycolysis by targeting LDH-A in mice intoxicated with $\mathrm{CCl}_{4}$. Animal grouping and treatments refer to the Methods section. a Measurements of hepatic lactate levels. $\mathbf{b}$ Real-time PCR analyses of mRNA expression of HK2, PFK1, PKM2 and LDH-A in liver tissues. $\mathbf{c}$ Western blot analyses of protein expression of HK2, PFK1, PKM2 and LDH-A in liver tissues with quantification. d, e Immunofluorescence analyses of HK2, PFK1, PKM2, and LDH-A in liver tissues. Staining with a-SMA was used to indicate HSCs, scale bar: $20 \mu \mathrm{m}$. For statistical significance of this figure: ${ }^{* *} p<0.01$ vs. control, ${ }^{* * *} p<0.001$ vs. control; ${ }^{\# \#} p<0.01$ vs. $\mathrm{CCl}_{4} ;{ }^{\circledR} p<0.05$ vs. $\mathrm{CCl}_{4}+$ oroxylin $\mathrm{A}+$ adenovirus vector, ${ }^{\&}{ }^{\&} p<0.01$ vs. $\mathrm{CCl}_{4}+$ oroxylin $\mathrm{A}+$ adenovirus vector

had lower abundance in the HSCs of oroxylin A-treated fibrotic mice compared to the model group, but overexpression of LDH-A impaired the effects of oroxylin A (Fig. 8d, e). We finally examined HSC contraction, and found that MLC2 phosphorylation was significantly increased in mouse fibrotic liver but was decreased by oroxylin A treatment; whereas overexpression of LDH-A rescued oroxylin A-inhibited MLC2 phosphorylation (Fig. 9a, b). Vimentin is a major component of cytoskeleton responsible for stabilization of cytoskeletal interactions [28], and is frequently used as a marker of cell contraction [29]. Here, immunofluorescence analysis of vimentin showed that HSC contraction was enhanced in mouse fibrotic liver but was inhibited by oroxylin A treatment; however, overexpression of LDH-A restored HSC contractile capacity in oroxylin A-treated fibrotic mice (Fig. 9b). Taken together, suppression of HSC glycolysis and contraction by oroxylin A contributed to the reduction of liver fibrosis in mice, and these effects were dependent on inhibition of LDH-A.

\section{Discussion}

HSCs are located in the space of Disse and contact closely with sinusoidal endothelial cells. The contractile phenotype of HSCs has been critically implicated in liver's response to various injuries, and the density and coverage of HSCs in the sinusoidal lumen are found to be increased during hepatic fibrosis [5]. It is recognized that the enhanced contractility of HSCs increases the resistance in sinusoidal blood flow and aggravates hepatic sinusoidal capillarization and remolding, leading to the development of portal hypertension, a highly lethal complication of advanced chronic liver disease [30]. Accordingly, restriction of HSC contraction represents a novel intervention strategy for liver fibrosis or cirrhosis, as well as portal hypertension. We recently reported that oroxylin A had significant antifibrotic and hepatoprotective effects in vitro and in vivo [17, 31, 32], and observed that oroxylin A could improve sinusoidal vascular remodeling [27]. These observations directed us to investigate whether modulation of HSC contractile phenotype was involved in oroxylin A's effects. Interestingly, our current data uncovered the association and identified regulation of aerobic glycolysis as a linking molecular event in oroxylin A' effects.

Accumulating evidence suggests that metabolic reprogramming controls the fate and transdifferentiation of 


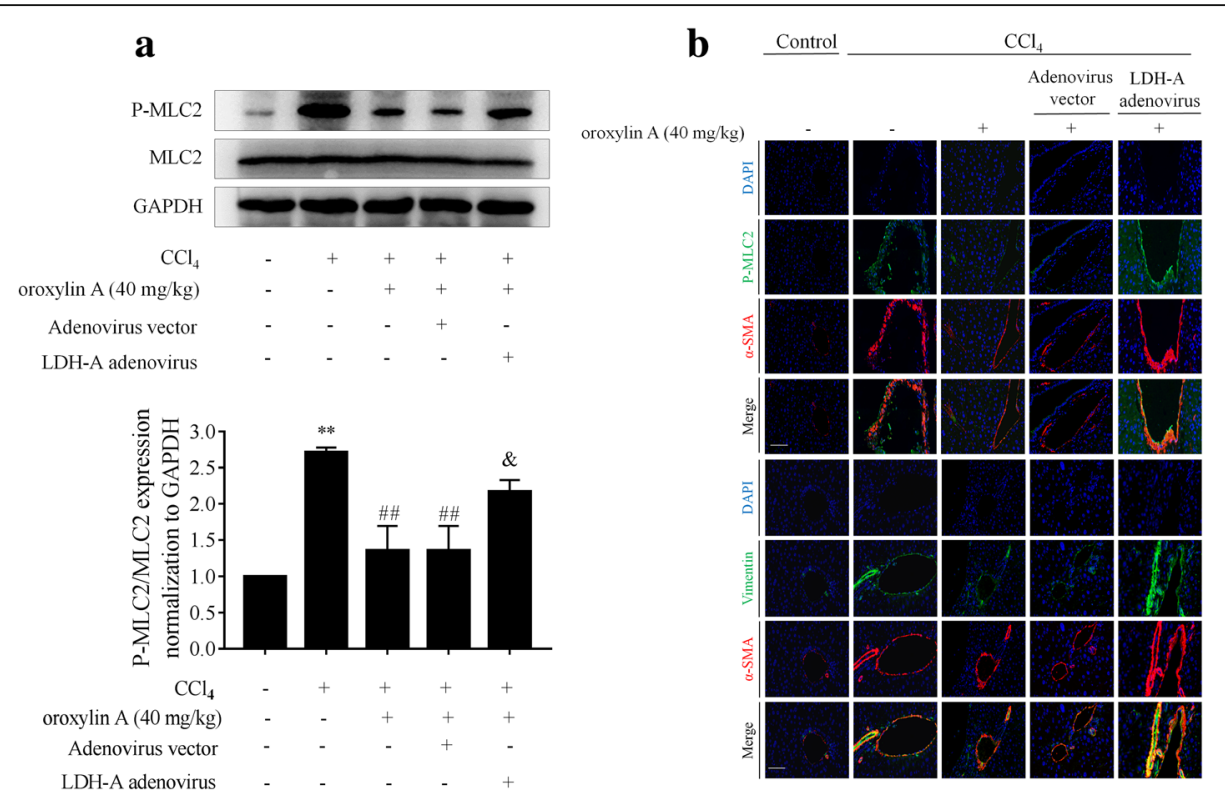

Fig. 9 Oroxylin A inhibits HSC contraction by targeting LDH-A in mice intoxicated with $\mathrm{CCl}_{4}$. Animal grouping and treatments refer to the Methods section. a Western blot analyses of MLC2 phosphorylation in liver tissues with quantification. $\mathbf{b}$ Immunofluorescence analyses of p-MLC2 and vimentin in liver tissues. Staining with a-SMA was used to indicate HSCs, scale bar: $20 \mu \mathrm{m}$. For statistical significance of this figure: ${ }^{* *} p<0.01$ vs. control; ${ }^{\# \#} p<0.01$ vs. $\mathrm{CCl}_{4} ;{ }^{\&} p<0.05$ vs. $\mathrm{CCl}_{4}+$ oroxylin $\mathrm{A}+$ adenovirus vector

HSCs, and is a conserved response to liver injury. Induction of aerobic glycolysis, similar to the Warburg effect described in tumor cells, has been proven to be a driving force of the dramatic phenotypic alterations of HSCs during hepatic repair, including the high proliferative and fibrogenic activities [12]. This phenomenon can be explained by the fact that glycolysis produces ATP at a faster rate than oxidative phosphorylation, although it only generates two ATP molecules per molecule of glucose. Glycolysis thus is a faster and shorter pathway for energy generation used by some cells to meet the high demands of rapid proliferation [33]. This metabolic switch has important therapeutic relevance and implication for liver fibrosis. Indeed, our previous work demonstrated that the well-known natural product curcumin inhibited HSC activation and reduced hepatic fibrosis through disrupting aerobic glycolysis [34, 35]. In current work, we postulated that the contractile phenotype of HSCs could also be governed by aerobic glycolysis and drug-induced metabolic perturbation could affect HSC contraction and related pathology in liver fibrosis. We found that oroxylin A potently inhibited HSC contraction evidenced by interruption of cytoskeleton arrangement and reduced MLC2 phosphorylation, and meanwhile, the glycolytic flux and activity were effectively blocked by oroxylin A evidenced by reduced glucose uptake and consumption, decreased lactate production and downregulation of three key rate-limiting enzymes. More importantly, we identified that oroxylin A blockade of aerobic glycolysis contributed to the restriction of HSC contraction. This point was easily understandable, because many components of the contraction machinery are involved in the efficient coupling of energy source and dependent on myosin-actin interaction using ATP [36]. The energy-contraction coupling was disrupted by blockade of aerobic glycolysis and reduction of energy supply in oroxylin-treated HSCs.

We subsequently investigated the potential upstream molecule mediating oroxylin A disruption of the energycontraction coupling machinery. We focused on the role of LDH-A because of the following points. (i) LDH-A was highly expressed in human fibrotic liver, implying a close association between LDH-A and hepatic fibrogenesis. (ii) LDH-A converts pyruvate, the final product of glycolysis, to lactate, shifting the use of glucose metabolites from simple energy production to acceleration of cell growth and replication, and thus LDH-A activity has been characterized as a promising target in cancer therapy by preventing cancer cells from proliferating [10]. (iii) LDH-A was newly recognized as a regulator of gene transcription via translocating into nucleus and binding to DNA, and phosphorylation of LDH at Tyr238 has been characterized to be important for its nuclear translocation [37]. Here, we observed that oroxylin A suppressed the expression and activity of LDH-A in HSCs, and, using chemical and genetic approaches, confirmed that inhibition of LDH-A was a prerequisite for oroxylin A reduction of glycolysis-dependent HSC contraction and liver fibrosis in vitro and in vivo. These results raised an interesting question that why modulation of LDH-A could be the causative event in this context given that LDH-A works at the final stage of 
glycolysis pathway. We postulated that this could be explained by two reasons. (i) Inhibition of LDH-A by oroxylin A synergistically blocked the glycolytic flux, leading to the reduced energy production and resultant restriction of contraction. (ii) LDH-A could regulate the expression of glycolysis rate-limiting enzymes such as HK2, PFK1 and PKM2. LDH-A might act as a transcription factor or co-activator to increase the transcription of these enzymes. This speculation could be, at least partially, supported by the observation that the de novo synthesis of these enzymes was inhibited by oroxylin A, blocking each rate-limiting step of glycolysis. We understand that our results could not rule out the possibility that the expression of these enzymes was inhibited by oroxylin A directly, or indirectly by targeting other molecules, given the fact that natural products commonly have multiple targets within cells.

\section{Conclusions}

In conclusion, our current work linked the aerobic glycolysis pathway to the contractile phenotype of HSCs, and uncovered that oroxylin A blocked glycolysis-dependent HSC contraction and reduced hepatic fibrosis through inhibition of LDH-A. We suggested LDH-A as a promising target for disruption of HSC metabolism implicated in liver fibrosis therapy.

\section{Additional files}

\section{Additional file 1: Table S1. Primer sequences for real-time PCR. (DOCX $16 \mathrm{~kb}$ ) \\ Additional file 2: Figure S1. Oroxylin A reduces the intracellular ATP levels in HSCs. LX2 cells were treated with oroxylin A at indicated concentrations for $24 \mathrm{~h}$. Measurements of intracellular ATP levels. For statistical significance of this figure: ${ }^{*} p<0.05 \mathrm{vs}$. control. (TIF $43 \mathrm{~kb}$ )}

Additional file 3: Figure S2. Effects of 2-DG or galloflavin cell viability using MTT assays. a LX2 cells were treated with 2-DG at indicated concentrations for $24 \mathrm{~h}$. b Hepatocyte LO2 cells were treated with 2-DG at indicated concentrations for $24 \mathrm{~h}$. c LX2 cells were treated with galloflavin at indicated concentrations for $24 \mathrm{~h}$. b Hepatocyte LO2 cells were treated with galloflavin at indicated concentrations for $24 \mathrm{~h}$. For statistical significance of this figure: ${ }^{*} p<0.05$ vs. control, ${ }^{* *} p<0.01$ vs. control. (TIF $68 \mathrm{~kb}$ )

Additional file 4: Figure S3. Real-time $P C R$ analyses of mRNA expression of LDH-A in human healthy liver or fibrotic liver. For statistical significance of this figure: * $p<0.05$ vs. healthy liver. (TIF 43 kb)

Additional file 5: Figure S4. LX2 cells were transfected with LDH-A overexpression plasmid and/or treated with oroxylin A at indicated concentrations for $24 \mathrm{~h}$. a Western blot analyses of protein expression of LDH-A. b Western blot analyses of protein expression of HK2, PFK1 and PKM2. (TIF $104 \mathrm{~kb}$ )

Additional file 6: Figure S5. LX2 cells were transfected with LDH-A overexpression plasmid and/or treated with oroxylin A at indicated concentrations for $24 \mathrm{~h}$. a Western blot analyses of MLC2 phosphorylation. b Cytoskeleton fluorescence staining, scale bar: $20 \mu \mathrm{m}$ (TIF 793 kb)

\section{Abbreviations}

2-DG: 2-Deoxy-D-glucose; ALT: Alanine aminotransferase; AST: Aspartate aminotransferase; $\mathrm{CCl}_{4}$ : Carbon tetrachloride; DAPI: Diamidino-phenyl-indole; ECAR: Extracellular acidification rate; GAPDH: Gglyceraldehyde phosphate dehydrogenase; GOD: Glucose oxidase; H\&E: Hematoxylin-eosin;
HA: Hyaluronic acid; HK2: Hexokinase 2; HSC: Hepatic stellate cell; Hyp: Hydroxyproline; IBIL: Indirect bilirubin; LDH: Lactate dehydrogenase; LN: Laminin; MLC2: Myosin light chain 2; PC-III: Procollagen type III; PFK1: Phosphofructokinase 1; PKM2: Pyruvate kinas type M2; SEM: Scanning electronic microscopy; TBIL: Total bilirubin; a-SMA: a-Smooth muscle actin

\section{Funding}

The National Natural Science Foundation of China (81870423, 31401210, 31571455, 31600653, and 81600483), the Open Project Program of Jiangsu Key Laboratory for Pharmacology and Safety Evaluation of Chinese Materia Medica (JKLPSE201815 and JKLPSE 201504), the Project of the Priority Academic Program Development of Jiangsu Higher Education Institutions (PAPD), and the Qing Lan Project of Jiangsu Province.

\section{Availability of data and materials}

All data generated or analyzed during this study are included in this published article.

\section{Authors' contributions}

Conception and design: FW, FZ and SZ. Data collection and interpretation: FW, YJ, ML, and LW. Providing materials and technique supports: QG, ST, and HD. Drafting of the manuscript: FW and FZ. Critical revision of the article for important intellectual content: JS, AC, and SZ. All authors have read and approved the final manuscript.

\section{Ethics approval}

Animal experimental procedures were approved by the Institutional and Local Committee on the Care and Use of Animals of Nanjing University of Chinese Medicine, and all animals were received humane care according to the National Institutes of Health (USA) guidelines. All protocols concerning the use of patient samples in this study were approved by the Medical Ethical Committee of the Nanjing Hospital Affiliated to Nanjing University of Chinese Medicine

\section{Consent for publication}

All authors agreed on the article.

\section{Competing interests}

The authors declare that they have no competing interests.

\section{Publisher's Note}

Springer Nature remains neutral with regard to jurisdictional claims in published maps and institutional affiliations.

\section{Author details}

'Jiangsu Key Laboratory for Pharmacology and Safety Evaluation of Chinese Materia Medica, School of Pharmacy, Nanjing University of Chinese Medicine, Nanjing 210023, China. ${ }^{2}$ Jiangsu Key Laboratory of Therapeutic Material of Chinese Medicine, School of Pharmacy, Nanjing University of Chinese Medicine, Nanjing 210023, China. ${ }^{3}$ State Key Laboratory Cultivation Base for TCM Quality and Efficacy, School of Pharmacy, Nanjing University of Chinese Medicine, Nanjing 210023, China. ${ }^{4}$ Jiangsu Key Laboratory of Carcinogenesis and Intervention, China Pharmaceutical University, Nanjing 210009, China. ${ }^{5}$ The Nanjing Hospital Affiliated to Nanjing University of Chinese Medicine, Nanjing 210003, China. ${ }^{6}$ Department of Pathology, School of Medicine, Saint Louis University, Saint Louis, MO 63104, USA

Received: 27 December 2018 Accepted: 4 February 2019

Published online: 11 February 2019

\section{References}

1. Friedman SL. Hepatic stellate cells: protean, multifunctional, and enigmatic cells of the liver. Physiol Rev. 2008;88:125-72.

2. Kisseleva T. The origin of fibrogenic myofibroblasts in fibrotic liver. Hepatology. 2017;65:1039-43.

3. Hellerbrand C. Hepatic stellate cells--the pericytes in the liver. Pflugers Arch. 2013;465:775-8.

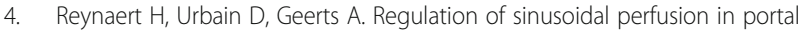
hypertension. Anat Rec (Hoboken). 2008;291:693-8.

5. Soon RK Jr, Yee HF Jr. Stellate cell contraction: role, regulation, and potential therapeutic target. Clin Liver Dis. 2008;12:791-803 viii. 
6. Tang DD, Gerlach $\mathrm{BD}$. The roles and regulation of the actin cytoskeleton, intermediate filaments and microtubules in smooth muscle cell migration. Respir Res. 2017;18:54.

7. Masters TA, Kendrick-Jones J, Buss F. Myosins: domain organisation, motor properties, physiological roles and cellular functions. Handb Exp Pharmacol. 2017:235:77-122.

8. Vander Heiden MG, Cantley LC, Thompson CB. Understanding the Warburg effect: the metabolic requirements of cell proliferation. Science. 2009;324: 1029-33.

9. Li XB, Gu JD, Zhou QH. Review of aerobic glycolysis and its key enzymes new targets for lung cancer therapy. Thorac Cancer. 2015;6:17-24.

10. Feng $Y$, Xiong $Y$, Qiao T, Li X, Jia L, Han Y. Lactate dehydrogenase a: a key player in carcinogenesis and potential target in cancer therapy. Cancer Med. 2018.

11. Di Stefano G, Manerba M, Di lanni L, Fiume L. Lactate dehydrogenase inhibition: exploring possible applications beyond cancer treatment. Future Med Chem. 2016;8:713-25.

12. Chen Y, Choi SS, Michelotti GA, Chan IS, Swiderska-Syn M, Karaca GF, Xie G, Moylan CA, Garibaldi F, Premont R, et al. Hedgehog controls hepatic stellate cell fate by regulating metabolism. Gastroenterology. 2012;143:1319-29 e11.

13. Hu Y, Yang Y, You QD, Liu W, Gu HY, Zhao L, Zhang K, Wang W, Wang XT, Guo QL. Oroxylin a induced apoptosis of human hepatocellular carcinoma cell line HepG2 was involved in its antitumor activity. Biochem Biophys Res Commun. 2006;351:521-7.

14. Yang Y, Hu Y, Gu HY, Lu N, Liu W, Qi Q, Zhao L, Wang XT, You QD, Guo QL. Oroxylin a induces G2/M phase cell-cycle arrest via inhibiting Cdk7mediated expression of Cdc2/p34 in human gastric carcinoma BGC-823 cells. J Pharm Pharmacol. 2008;60:1459-63.

15. Dai Q, Yin Q, Wei L, Zhou Y, Qiao C, Guo Y, Wang X, Ma S, Lu N. Oroxylin a regulates glucose metabolism in response to hypoxic stress with the involvement of hypoxia-inducible factor- 1 in human hepatoma HepG2 cells. Mol Carcinog. 2016;55:1275-89

16. Wei L, Zhou Y, Qiao C, Ni T, Li Z, You Q, Guo Q, Lu N. Oroxylin a inhibits glycolysis-dependent proliferation of human breast cancer via promoting SIRT3-mediated SOD2 transcription and HIF1alpha destabilization. Cell Death Dis. 2015;6:e1714.

17. Chen W, Zhang Z, Yao Z, Wang L, Zhang F, Shao J, Chen A, Zheng S. Activation of autophagy is required for Oroxylin a to alleviate carbon tetrachloride-induced liver fibrosis and hepatic stellate cell activation. Int Immunopharmacol. 2018;56:148-55.

18. Zhang F, Lu S, He J, Jin H, Wang F, Wu L, Shao J, Chen A, Zheng S. Ligand activation of PPARgamma by Ligustrazine suppresses Pericyte functions of hepatic stellate cells via SMRT-mediated Transrepression of HIF-1alpha. Theranostics. 2018:8:610-26.

19. Zhang F, Hao M, Jin H, Yao Z, Lian N, Wu L, Shao J, Chen A, Zheng S. Canonical hedgehog signalling regulates hepatic stellate cell-mediated angiogenesis in liver fibrosis. Br J Pharmacol. 2017;174:409-23.

20. Fu LH, Qi C, Lin J, Huang P. Catalytic chemistry of glucose oxidase in cancer diagnosis and treatment. Chem Soc Rev. 2018;47:6454-72.

21. Papkovsky DB, Zhdanov AV. Cell energy budget platform for assessment of cell metabolism. Methods Mol Biol. 2015;1265:333-48.

22. Zhang F, Zhang Z, Kong D, Zhang X, Chen L, Zhu X, Lu Y, Zheng S. Tetramethylpyrazine reduces glucose and insulin-induced activation of hepatic stellate cells by inhibiting insulin receptor-mediated PI3K/AKT and ERK pathways. Mol Cell Endocrinol. 2014;382:197-204.

23. Abreu-Blanco MT, Watts JJ, Verboon JM, Parkhurst SM. Cytoskeleton responses in wound repair. Cell Mol Life Sci. 2012;69:2469-83.

24. Hong F, Haldeman BD, Jackson D, Carter M, Baker JE, Cremo CR. Biochemistry of smooth muscle myosin light chain kinase. Arch Biochem Biophys. 2011;510:135-46.

25. Storey KB. Comparative enzymology-new insights from studies of an "old" enzyme, lactate dehydrogenase. Comp Biochem Physiol B Biochem Mol Biol. 2016;199:13-20

26. Fiume L, Vettraino M, Carnicelli D, Arfilli V, Di Stefano G, Brigotti M. Galloflavin prevents the binding of lactate dehydrogenase a to single stranded DNA and inhibits RNA synthesis in cultured cells. Biochem Biophys Res Commun. 2013;430:466-9.

27. Zhang C, Bian M, Chen X, Jin H, Zhao S, Yang X, Shao J, Chen A, Guo Q, Zhang $F$, et al. Oroxylin a prevents angiogenesis of LSECs in liver fibrosis via inhibition of YAP/HIF-1alpha signaling. J Cell Biochem. 2018;119:2258-68.
28. Styers ML, Kowalczyk AP, Faundez V. Architecture of the vimentin cytoskeleton is modified by perturbation of the GTPase ARF1. J Cell Sci. 2006;119:3643-54

29. Wang R, Li Q, Tang DD. Role of vimentin in smooth muscle force development. Am J Physiol Cell Physiol. 2006;291:C483-9.

30. Iwakiri Y. Pathophysiology of portal hypertension. Clin Liver Dis. 2014;18: 281-91.

31. Jin H, Lian N, Bian M, Zhang C, Chen X, Shao J, Wu L, Chen A, Guo Q, Zhang $F$, et al. Oroxylin a prevents alcohol-induced hepatic steatosis through inhibition of hypoxia inducible factor 1alpha. Chem Biol Interact. 2018:285:14-20

32. Jin H, Lian N, Bian M, Zhang C, Chen X, Shao J, Wu L, Chen A, Guo Q, Zhang $F$, et al. Oroxylin a inhibits ethanol-induced hepatocyte senescence via YAP pathway. Cell Prolif. 2018,51:e12431.

33. Pfeiffer T, Schuster S, Bonhoeffer S. Cooperation and competition in the evolution of ATP-producing pathways. Science. 2001;292:504-7.

34. Lian N, Jiang Y, Zhang F, Jin H, Lu C, Wu X, Lu Y, Zheng S. Curcumin regulates cell fate and metabolism by inhibiting hedgehog signaling in hepatic stellate cells. Lab Investig. 2015;95:790-803.

35. Lian N, Jin H, Zhang F, Wu L, Shao J, Lu Y, Zheng S. Curcumin inhibits aerobic glycolysis in hepatic stellate cells associated with activation of adenosine monophosphate-activated protein kinase. IUBMB Life. 2016;68: 589-96.

36. Takashima S. Phosphorylation of myosin regulatory light chain by myosin light chain kinase, and muscle contraction. Circ J. 2009;73:208-13.

37. Boukouris AE, Zervopoulos SD, Michelakis ED. Metabolic enzymes moonlighting in the nucleus: metabolic regulation of gene transcription. Trends Biochem Sci. 2016;41:712-30.

\section{Ready to submit your research? Choose BMC and benefit from:}

- fast, convenient online submission

- thorough peer review by experienced researchers in your field

- rapid publication on acceptance

- support for research data, including large and complex data types

- gold Open Access which fosters wider collaboration and increased citations

- maximum visibility for your research: over $100 \mathrm{M}$ website views per year

At BMC, research is always in progress.

Learn more biomedcentral.com/submissions 